\title{
Programmable Mechanical Metamaterials
}

\author{
Bastiaan Florijn, ${ }^{*}$ Corentin Coulais, ${ }^{\dagger}$ and Martin van Hecke ${ }^{\ddagger}$ \\ Huygens-Kamerling Onnes Lab, Universiteit Leiden, Postbus 9504, 2300 RA Leiden, The Netherlands
}

(Received 15 July 2014; published 24 October 2014)

\begin{abstract}
We create mechanical metamaterials whose response to uniaxial compression can be programmed by lateral confinement, allowing monotonic, nonmonotonic, and hysteretic behavior. These functionalities arise from a broken rotational symmetry which causes highly nonlinear coupling of deformations along the two primary axes of these metamaterials. We introduce a soft mechanism model which captures the programmable mechanics, and outline a general design strategy for confined mechanical metamaterials. Finally, we show how inhomogeneous confinement can be explored to create multistability and giant hysteresis.
\end{abstract}

DOI: 10.1103/PhysRevLett.113.175503

PACS numbers: 81.05.Zx, 46.70.De, 62.20.mq, 81.05.Xj

Metamaterials derive their unusual properties from their structure, rather than from their composition [1]. Important examples of mechanical metamaterials are auxetic (negative Poisson's ratio) materials [2], materials with vanishing shear modulus [3-5], materials with negative compressibility $[6,7]$, singularly nonlinear materials [8,9], and topological metamaterials [10-12]. Of particular recent interest are mechanical metamaterials whose functionality relies on elastic instabilities, such as quasi-2D slabs perforated with a square array of holes [13-17]. When compressed, these "holey sheets" undergo a bucklinglike pattern transformation, which can be explored to obtain switchable auxetics [15], chiral and phononic properties [18-20], and 3D "buckliballs" [21,22]. An important limitation common to all these metamaterials is that each mechanical functionality requires a different structure.

Here we present a novel strategy to create programmable mechanical metamaterials, where the response of a single structure is determined by confinement. The core idea is illustrated in Fig. 1 for a biholar sheet, a quasi-2D elastic slab of material patterned by a regular array of large and small holes. The difference in hole sizes breaks one of the $90^{\circ}$ rotation symmetries which is present for equal hole sizes. This causes a difference in the polarization of the hole pattern, depending on whether $x$ compression or $y$ compression is dominant-see Figs. 1(c)-1(d) [23].

By constraining this metamaterial in the $x$ direction, and then compressing it in the $y$ direction, the material undergoes a pattern switch from an $x$-polarized to a $y$-polarized state, as illustrated in Figs. 1(c)-1(d). Depending on the magnitude of the $x$ confinement, this pattern switch can be either smooth or discontinuous, and the force-displacement curves for the $y$ deformations can be tuned from monotonic to nonmonotonic (unstable) to hysteretic - all for a single biholar sheet.

We observe this tunability in experiments on systems of various sizes as well as in numerical simulations of the unit cell-this is a robust phenomenon. We introduce a simple model that qualitatively captures all these different mechanical behaviors, and which allows a precise study of the bifurcation scenario that underlies this phenomenology. Finally, we show that the sensitive nature of the hysteretic switching between $x$ - and $y$-polarized patterns can be explored in larger systems, where controlled inhomogeneities lead to multistability and giant hysteresis. We suggest that confinement is a general mechanism for a
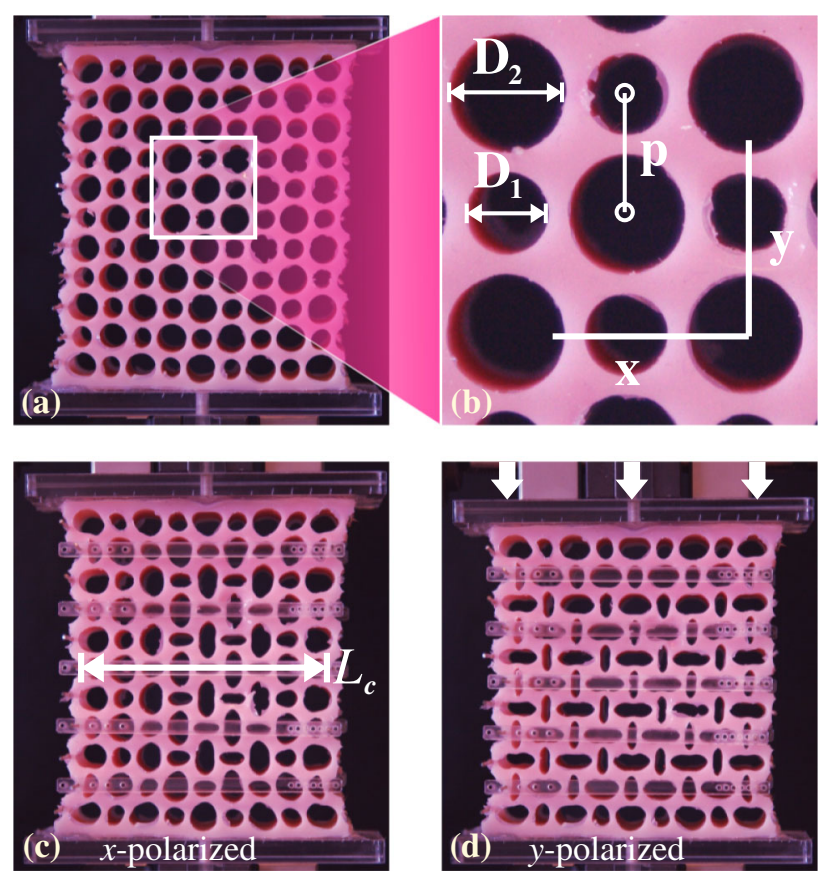

FIG. 1 (color online). (a)-(b) A biholar sheet characterized by hole diameters $D_{1}=10 \mathrm{~mm}, D_{2}=7 \mathrm{~mm}$, and pitch $p=10 \mathrm{~mm}$. (c) Uniform confinement $L_{c}$ in the $x$ direction (by semitransparent clamps) leads to an $x$ polarization of the material. (d) Sufficient $y$ compression of this $x$-confined material leads to a switch of the polarization, and concomitant nontrivial mechanical behavior. Without $x$ confinement the pattern will look very similar. 

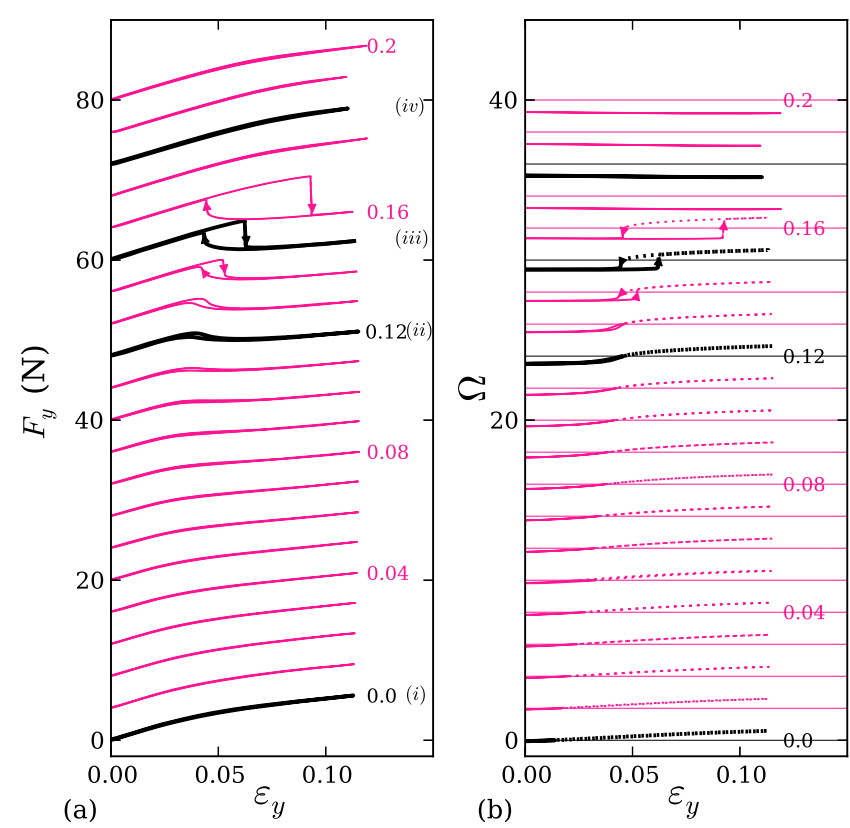

FIG. 2 (color online). Mechanical response of a $5 \times 5$ sheet with $D_{1}=10 \mathrm{~mm}, D_{2}=7 \mathrm{~mm}(\chi=0.3, t=0.15)$ for increasing confinement $\varepsilon_{x}$ [24]. Curves are offset for clarity. (a) The force curves $F_{y}\left(\varepsilon_{y}\right)$ show monotonic, nonmonotonic, and hysteretic behavior. The curves labeled (i)-(iv) correspond to four qualitatively different behaviors. (b) Polarization of the large center hole, where full (dashed) curves indicate negative (positive) values. For movies of case (i)-(iv), see [25].

much larger class of programmable mechanical metamaterials, and outline how our model opens a pathway towards rational design of these materials.

Experimental setup and sample preparation.-We use quasi-2D elastic sheets of thickness $35 \mathrm{~mm}$ to avoid outof-plane buckling. Their 2D structure is shown in Fig. 1. The dimensionless numbers that characterize their geometry are the biholarity, $\chi:=\left|D_{1}-D_{2}\right| / p$, and the dimensionless thickness of their most slender parts, $t=1-(D 1+D 2) / 2 p$. To create these biholar sheets, we pour a two component silicone elastomer (Zhermack Elite Double 8, Young's Modulus $E \simeq 220 \mathrm{kPa}$, Poisson's ratio $\nu \simeq 0.5$ ) in a mold that consists of brass cylinders of diameter $D_{1}$ and $D_{2}$, precisely placed in the square grid of two laser-cut sidewalls with $p=10 \mathrm{~mm}$. We cut the sheets lateral sides and glue their flat bottom and top to Plexiglas plates, and then probe their mechanical response in a uniaxial testing device (Instron type 5965), which controls the compressive strain $\varepsilon_{y}$ better than $10^{-5}$ and allows us to measure the compressive force $F_{y}$ with an accuracy $10^{-4} \mathrm{~N}$.

To impose confinement we glue copper rods of diameter $1.2 \mathrm{~mm}$ on the sides of our material, see Fig. 1, and use laser cut plastic clamps which have holes at distance $L_{c}$ to exert a fixed $x$ strain, which we approximate as $\varepsilon_{x}:=1-L_{c} / N p$, where $N$ is the number of holes per row [24]. We image the deformations of the material with a CCD camera, resulting in a spatial resolution of $0.03 \mathrm{~mm}$, and track the position and shape of the holes. This allows us to determine the polarization of the holes, defined as $\Omega:= \pm\left(1-p_{2} / p_{1}\right) \cos 2 \phi$, where $p_{1}\left(p_{2}\right)$ is the major (minor) axis and $\phi$ the angle between the major axis and $x$ axis-as the values of $\phi$ of the small and big holes are different, we define the sign of $\Omega$ such that $\Omega$ is positive (negative) for $y$ polarization ( $x$ polarization) [23].

Programmable mechanical response.-As we will see, inhomogeneities play an important role for large systems, so to probe the mechanics of asymptotically large homogeneous materials, we start our exploration with the smallest experimentally feasible building block, a $5 \times 5$ biholar sheet.

In Fig. 2 we present the force $F_{y}\left(\varepsilon_{y}\right)$ and polarization $\Omega\left(\varepsilon_{y}\right)$ of the large center hole for a range of values of $\varepsilon_{x}$. We can distinguish four qualitatively different types of mechanical response. (i) For $\varepsilon_{x} \lesssim 0.09$, the force increases monotonically with $\varepsilon_{y}$ and the polarization smoothly grows from its initial negative value to positive values. (ii) For $0.10 \lesssim \varepsilon_{x} \lesssim 0.13, F_{y}\left(\varepsilon_{y}\right)$ becomes nonmonotonic-here the material has negative uniaxial compressibility. The increase in polarization gets focused in the "negative slope" regime but remains monotonic. (iii) For $0.14 \lesssim \varepsilon_{x} \lesssim 0.16$, $F_{y}\left(\varepsilon_{y}\right)$ exhibits a hysteretic transition with a corresponding hysteretic switch from $x$ to $y$ polarization. Note that in this case, the polarization is nonmonotonic and initially decreases-hence, $y$ compression makes the center hole initially more $x$ polarized. (iv) For $\varepsilon_{x} \gtrsim 0.17$ the material becomes increasingly strongly $x$ polarized and does no longer switch to $y$ polarization and $F_{y}\left(\varepsilon_{y}\right)$ is smooth and monotonic. We note that in this case, additional experiments reveal that initial compression in the $y$ direction followed by $x$ confinement brings the material to a strongly $y$-polarized state. Hence, for strong biaxial confinement there are two stable states that are so different that uniaxial compression is not sufficient to make them switch.

We have explored this scenario for a number of different biholar sheets, and find the same four types of responses, provided that $\chi \lesssim 0.10$ and $t \gtrsim 0.15$ [26]. Moreover, as we will discuss below, such behavior is also found in larger systems. Hence, we conclude that confined biholar sheets can be tuned to exhibit four different types of mechanical behavior.

Numerics.-To probe whether these phenomena are robust, we have performed 2D plane strain finite element simulations (Abaqus) of a Neo-Hookean material on a $2 \times 2$ unit cell using periodic boundary conditions (Fig. 3). To probe numerically the various equilibrium branches, hysteresis and bistability, we use two different protocolsin protocol $A$ we first apply a confinement $\varepsilon_{x}$ and then a compression $\varepsilon_{y}$, whereas in protocol $B$ we first apply a large compression in the $y$ direction, then a confinement $\varepsilon_{x}$ and finally decompress in the $y$ direction until we reach $\varepsilon_{y}$. As shown in Fig. 3, these simulations exhibit the four types 

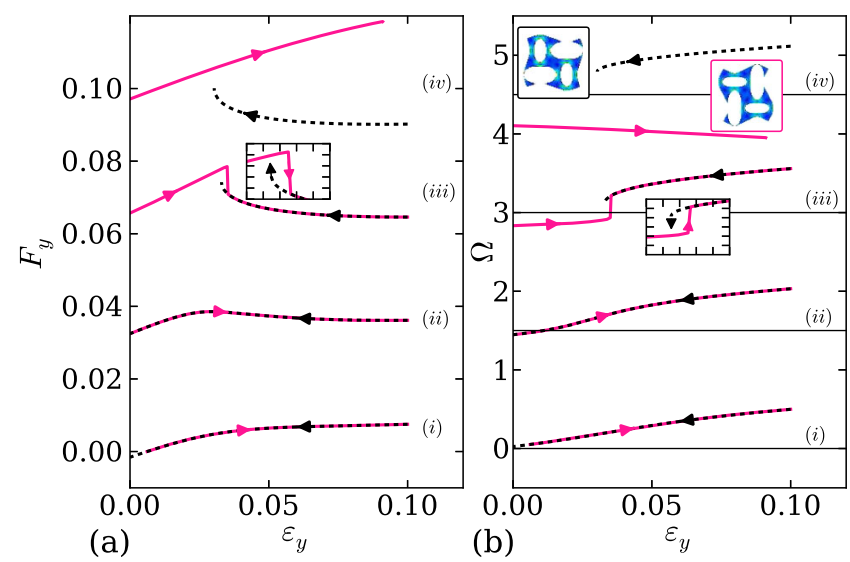

FIG. 3 (color online). Finite element simulations on a unit cell with periodic boundary conditions and $\chi=0.3, t=0.15$. (a) Force $F_{y}$ and (b) polarization $\Omega$, for (i) $\varepsilon_{x}=-1.0 \times 10^{-2}$, (ii) $\varepsilon_{x}=2.8 \times 10^{-2}$, (iii) $\varepsilon_{x}=3.4 \times 10^{-2}$, (iv) $\varepsilon_{x}=7.5 \times 10^{-2}$. Full (dashed) curves correspond to protocol $A(B)$. For a movie of case (iii), see [25].

of behavior (i)-(iv) observed in experiments. Moreover, these simulations reveal that in case (iv), the $x$ - and $y$ polarized branches become disconnected, consistent with our experimental data. The correspondence between experiments and simulations on systems with periodic boundaries show that our findings represent robust, bulktype behavior, and suggest that biholar metamaterials of arbitrary size remain functional.

Soft mechanism.-To qualitatively understand the mechanics of confined biholar sheets, we note that when $t \rightarrow 0$, the materials low energy deformations are equivalent to that of a mechanism of rigid rectangles coupled by hinges located at the necks of the "beams" (Fig. 4). The state of this mechanism is described by a single degree of freedom, $\theta$, which determines the internal dimensions $x_{i}$ and $y_{i}$. To model the storing of elastic energy, we couple this mechanism to outside walls at spacing $x_{o}$ and $y_{o}$ via a set of linear springs with zero rest length and spring constant $1 / 2$ [Fig. 4(c)]. As Figs. 4(a)-4(b) show, such a simple model qualitatively captures the full experimentally and numerically observed scenario, when we identify the clamping $\varepsilon_{x}$ with $1-x_{o}$ and compression $\varepsilon_{y}$ with $1-y_{o}$ [Figs. 4(a)-4(b)].

A geometric interpretation of the various equilibria and their bifurcations as $\varepsilon_{x}$ and $\varepsilon_{y}$ are varied provides much insight [25]. As illustrated in Fig. 4(d), the relation between the $x_{i}$ and $y_{i}$ can be represented as a smooth curve, which we refer to as $M$ (for mechanism). For given $\left(x_{o}, y_{o}\right)$, the elastic energy $E$ equals $\left(x_{i}-x_{o}\right)^{2}+\left(y_{i}-y_{o}\right)^{2}$, so that equienergy curves are circles of radius $E^{1 / 2}$. Stable (unstable) equilibria thus correspond to points on $M$ tangent to such circles, where $R_{M}$, the radius of curvature of $M$, is smaller (larger) than $E^{1 / 2}$. The experimental protocol varies $y_{o}$ at fixed $x_{o}$. Repeating this geometric construction while varying $y_{o}$ provides the corresponding stable and unstable equilibria, their elastic energies, and the force $F_{y}:=\partial_{y_{o}} E$.
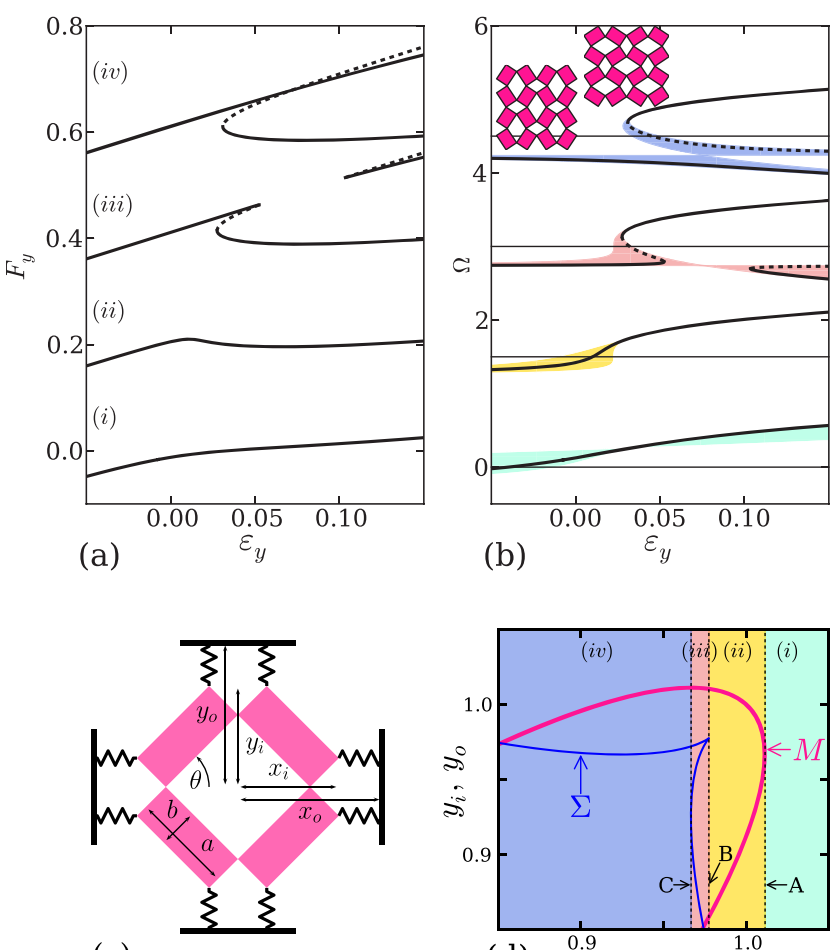

(c)

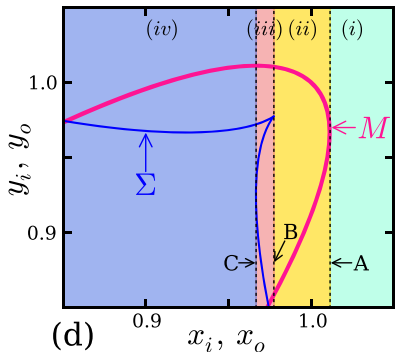

FIG. 4 (color online). (a)-(b) The force and polarization in our mechanical model for $\chi=0.3(a \approx 0.81, b \approx 0.6)$ exhibit behaviors (i)-(iv)- here full (dashed) curves correspond to stable (unstable) equilibria, and the insets show a $y$-and $x$-polarized state. The colored regions in (b) are obtained by sweeping $x_{0}$ through the ranges (i)-(iv) and tracing out the corresponding polarization curves. (c) Soft mechanism, with $\chi=2(a-b) /$ $(a+b)$, and where the undeformed configuration corresponds to $\theta=\pi / 4$, so that $a+b=\sqrt{2}$ (d) $M$ curve (thick solid pink) $\left(M:\left[\left(x_{i}-y_{i}\right) /(a-b)\right]^{2}+\left[\left(x_{i}+y_{i}\right) /(a+b)\right]^{2}=2\right)$, evolute $\Sigma$ (thin solid blue), and transitions $A-C$ between domains (i)-(iv). For movies illustrating case (i)-(iv) and $A-C$ see [25].

We now explore this model to understand the transition $A$ from monotonic to nonmonotonic force curves, the transition $B$ that leads to hysteresis, and the transition $C$, where the differently polarized branches become separated. In Fig. 4(d) we indicate the four trajectories corresponding to the data shown in Figs. 4(a)-4(b), as well as three trajectories labeled $A, B$, and $C$ that correspond to marginal curves which separate scenarios (i)-(iv). To understand the transitions $B$ and $C$, we will consider the evolute $\Sigma$, the locus of all the centers of curvature of $M$ [27]. When $\left(x_{o}, y_{o}\right)$ crosses $\Sigma$, saddle-node bifurcations occur-when $\left(x_{o}, y_{o}\right)$ crosses $\Sigma$ in a nongeneric manner, more complex bifurcations may arise [28].

Figure 4(d) now gives a clear geometric interpretation of the three transitions: $A$ : Curve $A$ is tangent to $M$, so that here the energy is purely quartic in $y_{o}$, and $\partial_{y} F_{y}=0$. Curve $A$ thus separates (i) monotonic force curves at larger $x_{0}$ from (ii) nonmonotonic force curves for smaller $x_{0}$. $B$ : Curve $B$ intersects the cusp of $\Sigma$, leading to a pair of 

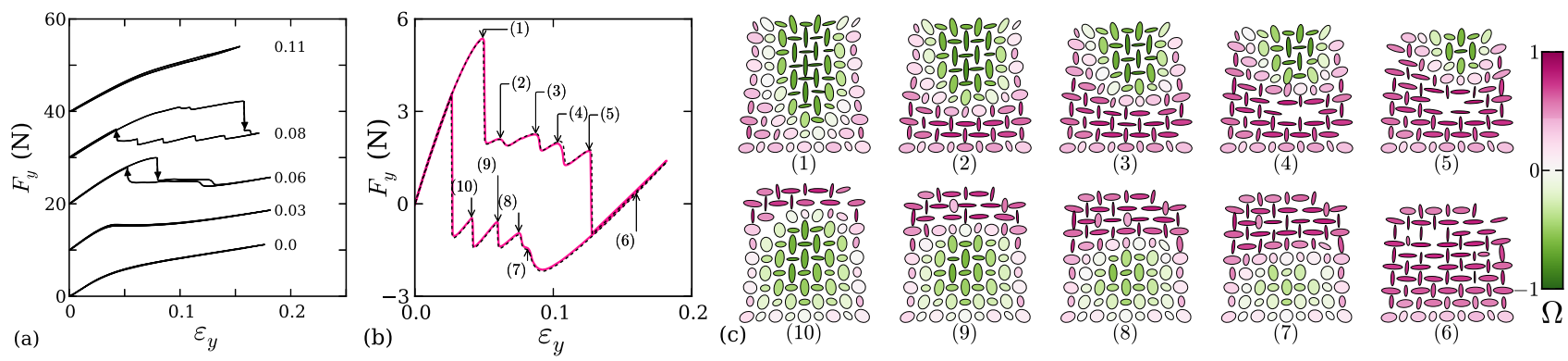

FIG. 5 (color online). (a) Force-displacement curves for a $9 \times 11$ sheet $\left(D_{1}=10 \mathrm{~mm}, D_{2}=7 \mathrm{~mm}, \chi=0.3, t=0.15\right)$ with horizontal confinement $\varepsilon_{x}$ as indicated exhibits behavior (i)-(iv) as well as multistable behavior. Curves are offset for clarity. (b) Forcedisplacement curve for an inhomogeneously confined $9 \times 11$ system, showing two giant hysteresis loops with multiple, perfectly reproducible polarization switching events. (c) Snapshots of the state of our experimental metamaterial corresponding to (1)-(10), where color codes for polarization.

saddle-node bifurcations which become separated for smaller $x_{o}$, and thus spawn a hysteresis loop. Curve $B$ thus separates case (ii) and (iii). $C$ : Finally, curve $C$ is tangent to $\Sigma$, which corresponds to a transcritical bifurcation where two solutions cross and exchange stability. As a result, for smaller $x_{o}$, the differently polarized branches decouple [Fig. 4(b)](iv). Curve $C$ thus separates (iii) and (iv), which can be seen as unfoldings of this transcritical bifurcation. For movies illustrating the geometrical construction for cases (i)-(iv) as well as $A-C$, see [25].

Larger systems. - To show that our observed mechanical functionality can be experimentally realized in larger systems, we show in Fig. 5(a) examples of $F_{y}\left(\varepsilon_{y}\right)$ for a $9 \times 11$ sheet. We observe the same four regimes as in Fig. 2 when the confinement is increased, illustrating the robustness of these phenomena. The main difference with smaller systems is that the hysteretic range is expanded and the force signal then has a complex structure exhibiting several peaks. These correspond to multiple switching events, due to inhomogeneities-as hysteresis corresponds to instability, even small inhomogeneities are amplified.

We exploit this sensitivity to inhomogeneities to create multistable states. We use five clamps of decreasing length as function of row number, corresponding to strains $\varepsilon_{x}=0.08$, $0.11, \ldots, 0.20$. As shown in Fig. 5(b), this results in a giant hysteresis loop with multiple peaks. We stress that Fig. 5(b) overlays two subsequent hysteretic loops, illustrating that this complex behavior is well reproducible. As shown in Fig. 5(c) and in [25], each of these peaks corresponds to a polarization switch of part of the material: Under compression and decompression, a polarization wave travels through our material. We note that the spatial configurations in the downsweep of $\varepsilon_{y}$ are not the same as in the upsweep, which can be understood from the observation that the most confined part of the system shows the most hysteresis.

Hence, inhomogeneous confinement provides an avenue for the realization of complex multistable systems. Moreover, these states with large hysteresis can also be seen as very effective dissipators of work, leading to novel strategies for mechanical damping [6,29].
Outlook.-We have introduced a class of programmable mechanical metamaterials whose functionality rests on two pillars: First, confinement allows us to store and release elastic energy, crucial for complex mechanics; second, a broken symmetry leads to competition and coupling between a secondary confinement and a primary strain.

The soft mechanism model suggests how to rationally design mechanical metamaterials with confinement controlled response. First, determine the bifurcation scenario when $\varepsilon_{x}$ is varied. Second, construct an evolute $\Sigma$ that is consistent with these sequence of bifurcations. The $M$ curve can then explicitly be constructed as the involute of $\Sigma$ [30]. Third, design a physical mechanism that possesses this $M$ curve; in principle any $M$ curve is encodable in a mechanism [31]. Finally, translate the rigid mechanism and hinges to a soft metamaterial with slender elements. Important work for the future is to explicitly demonstrate the feasibility of this design strategy [32].

Finally, our work leads to several open questions, of which we highlight three. First, for large, inhomogenous and multistable systems, how many distinct states can be reached when more complex parameter sweeps are allowed? Second, can we connect the mechanics of biholar sheets to the well-studied holey sheets with equal hole sizes, for which the mechanical response is not fully understood [13-19]? Third, can we use the strategies outlined here to create fully 3D mechanical metamaterials?

We acknowledge discussions with K. Bertoldi, K. Kamrin, P. Reis, and S. Waitukaitis. We thank $\mathrm{H}$. Imthorn, J. van Driel, and K. de Reus for exploratory experiments. B. F., C. C., and M. v. H. acknowledge funding from the Netherlands Organization for Scientific Research NWO.

\footnotetext{
florijn@physics.leidenuniv.nl †coulais@physics.leidenuniv.nl †hecke@physics.leidenuniv.nl
} 
[1] M. Kadic, T. Bückmann, R. Schittny, and M. Wegener, Rep. Prog. Phys. 76, 126501 (2013).

[2] R. Lakes, Science 235, 1038 (1987).

[3] G. W. Milton, J. Mech. Phys. Solids 40, 1105 (1992).

[4] M. Kadic, T. Bückmann, N. Stenger, M. Thiel, and M. Wegener, Appl. Phys. Lett. 100, 191901 (2012).

[5] T. Bückmann, M. Thiel, M. Kadic, R. Schittny, and M. Wegener, Nat. Commun. 5, 4130 (2014).

[6] R. S. Lakes, T. Lee, A. Bersie, and Y.C. Wang, Nature (London) 410, 565 (2001).

[7] Z. G. Nicolaou and A. E. Motter, Nat. Mater. 11, 608 (2012).

[8] M. Wyart, H. Liang, A. Kabla, and L. Mahadevan, Phys. Rev. Lett. 101, 215501 (2008).

[9] L. R. Gomez, A. M. Turner, M. van Hecke, and V. Vitelli, Phys. Rev. Lett. 108, 058001 (2012).

[10] C. L. Kane and T. C. Lubensky, Nat. Phys. 10, 39 (2014).

[11] B. G. Chen, N. Upadhyaya, and V. Vitelli, arXiv:1404.2263.

[12] J. Paulose, B. G. Chen, and V. Vitelli, arXiv:1406.3323.

[13] T. Mullin, S. Deschanel, K. Bertoldi, and M. C. Boyce, Phys. Rev. Lett. 99, 084301 (2007).

[14] K. Bertoldi, M. Boyce, S. Deschanel, S. Prange, and T. Mullin, J. Mech. Phys. Solids 56, 2642 (2008).

[15] K. Bertoldi, P. M. Reis, S. Willshaw, and T. Mullin, Adv. Mater. 22, 361 (2010).

[16] J. T. B. Overvelde, S. Shan, and K. Bertoldi, Adv. Mater. 24, 2337 (2012).

[17] J. Shim, S. Shan, A. Košmrlj, S. Kang, E. R. Chen, J. Weaver, and K. Bertoldi, Soft Matter 9, 8198 (2013).

[18] L. Wang and K. Bertoldi, Int. J. Solids Struct. 49, 2881 (2012).
[19] P. Wang, J. Shim, and K. Bertoldi, Phys. Rev. B 88, 014304 (2013).

[20] P. Wang, F. Casadei, S. Shan, J. C. Weaver, and K. Bertoldi, Phys. Rev. Lett. 113, 014301 (2014).

[21] J. Shim, C. Perdigou, E. R. Chen, K. Bertoldi, and P. M. Reis, Proc. Natl. Acad. Sci. U.S.A. 109, 5978 (2012).

[22] S. Babaee, J. Shim, J. C. Weaver, E. R. Chen, N. Patel, and K. Bertoldi, Adv. Mater. 25, 5044 (2013).

[23] Note that the major axis of the large ellipses is in the $x$ direction for $y$-polarized systems.

[24] We only clamp rows that end in small holes, to avoid subtle boundary effects [26]. As a result, the averaged horizontal strain is smaller than the control parameter $\epsilon_{x}$ (Fig. 1).

[25] See Supplemental Material at http://link.aps.org/ supplemental/10.1103/PhysRevLett.113.175503 for movies and their description.

[26] B. Florijn, C. Coulais, and M. van Hecke (unpublished).

[27] http://en.wikipedia.org/wiki/Evolute.

[28] J. Guckenheimer and P. Holmes, Nonlinear Oscillations, Dynamical Systems, and Bifurcations of Vector Fields, Applied Mathematical Sciences (Springer, New York, 1983), Vol. 42, ISBN 9780387908199.

[29] R. S. Lakes, Phys. Rev. Lett. 86, 2897 (2001).

[30] http://en.wikipedia.org/wiki/Involute.

[31] J. O'Rourke, How to Fold It: The Mathematics of Linkages, Origami and Polyhedra (Cambridge University Press, New York, 2011), ISBN 9781139498548.

[32] A. Jain, A. Bollinger, and T. M. Truskett, AIChE J. 60, 2732 (2014). 